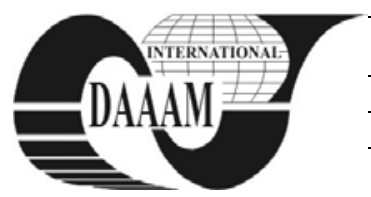

Annals of DAAAM for 2011 \& Proceedings of the 22nd International DAAAM Symposium, Volume 22, No. 1, ISSN 1726-9679 ISBN 978-3-901509-83-4, Editor B. Katalinic, Published by DAAAM International, Vienna, Austria, EU, 2011 Make Harmony between Technology and Nature, and Your Mind will Fly Free as a Bird Annals \& Proceedings of DAAAM International 2011

\title{
EXPERIMENTAL VALIDATION OF FEM FOR FRICTIONAL CONTACTS
}

\author{
PISCAN, I[uliana]; JANSSENS, T[hierry] \& PREDINCEA, N[icolae]
}

\begin{abstract}
Experimental validation of FEM is required to assure accurate results and to be able to update FE models, such that their simulation can be trusted and used in design. Here, the correlation between experimental results, coming from a flat on flat frictional contact on a tribometer, with its simulation in Ansys Workbench is discussed and validated. The validation is performed for two commonly used materials, viz. PVC and Al, and the normal load and displacement dependency of the frictional behaviour is also taken into account. It is shown that, by using appropriate inputs and an optimised representation of the non-linear frictional behaviour, an accurate frictional contact simulation is obtained
\end{abstract}

Key words: friction, contact, FEM, normal load, displacement

\section{INTRODUCTION}

Friction is unavoidably present in many mechanical systems. Their performance is considerably influenced by contact stiffness parameters which are determining the behaviour of their intrinsic connections such as flat on flat contacts or bolted connections. Stiffness effects on the performance of mechanical systems are due to the influence of deformations on static and fatigue strength, wear resistance, efficiency (friction losses), accuracy, dynamic/vibration stability, and manufacturability (Rivin, 1999). In function of its application, friction can be desirable e.g. in brakes, clutches and friction drives or undesirable in bearings, slides and joints. In both cases, it is important to be able to characterize and control the frictional behaviour in order to assure a good performance of the system.

The study of contact problems with friction has been the subject of numerous experimental investigations for hundreds of years and recently numerous computational investigations have been performed. One of the first investigations concerns a plane contact problem in full sliding (Muskhelishvili, 1966) in which the frictional stress is linearly proportional to the contact pressure. A frictional contact with stick and slip is considered in Galin, 1980. In numerous practical cases the frictional stress is a nonlinear function of pressure.

Although expansive research has been done, there are still many unknowns related to friction phenomena. In dry sliding contacts, the friction coefficient depends on various factors such as the applied load, surface topography, sliding velocity, temperature, elastic and plastic properties. The dependence of the friction coefficient on these factors is treated in numerous technical journals (Bengisu \& Akay, 1997; Kalker, 1975). The research elaborated by Bowden and Tabor, 1964 had an important role in the study of friction. They demonstrated that the static friction between two sliding surfaces is significantly influenced by the real area of contact. Their general conclusion was that the friction force consists of forces due to adhesion and mechanical deformations.

The characterization of friction depends significantly on the accuracy of experimental measurements. The frictional behaviour is generally a characteristic of the system, and not only of the materials in contact, therefore no single test can describe all types of frictional situations (Blau, 2001).
Furthermore, studies have been made in relating the friction coefficient to contact parameters and relevant operating conditions through theoretical modelling (Greenwood \& Tripp, 1967; Greenwood \& Williamson, 1966) though the most significant sources of guidance to practical values are experimental investigations.

\section{FRICTIONAL CONTACTS IN FEM}

The formulations of contact elements within FEM were developed in the 1970's. In our days, commercial FE-programs use contact models such as node to node which are typically used to model point-to-point contact applications, node to surface, which connects the nodes of the surface to the elements of the other surface. In industrial applications (seals, metalforming processes), due to the occurrence of large deformations, non-linear behaviour and large relative motion between the participating contact members a surface to surface formulation is advised to be used.

The main advantages of a 3D-FE model are (1) the proper utilization of correct geometry (dimensions, surface topography, degrees of freedom) and (2) the ability to calculate stresses and deformations in the entire body. 3D-FE models have a major disadvantage. Due to the very fine mesh they require high processing times for rough surfaces.

With the increased capabilities of digital computers, numerical methods became the main focus of researchers. Several formulations have been proposed to treat frictional contact problems using the Finite Element Method (FEM) (Karnopp, 1985; Kikuchi \& Oden, 1988).

Contact problems in FEM programs consist of two general classes: rigid-to-flexible and flexible-to-flexible. The flexibleto-flexible is the more common contact problem. In this case, both (or all) contacting bodies are deformable (i.e., have similar stiffness). An example of a flexible-to-flexible contact is bolted connections.

The frictional contact problem involves two distinct states: slip and stick. In the fundamental Coulomb friction model, two contacting surfaces can undergo the tangential force to a value of the magnitude along their contact interface before starting to slide. This state is known as sticking $F_{t}<\mu \cdot F_{n}$. In the Coulomb friction model an equivalent tangential force $F_{t}$ is defined, where the sliding on the contact surface begins as a fraction of the normal load $F_{n}, F_{t}=\mu \cdot F_{n}$, where $\mu$ represents the friction coefficient, defined as a material property. Sliding between the two surfaces occurs when the tangential force is exceeded $F_{t}>$ $\mu \cdot F_{n}$. Fig. 1 . represents the Coulomb frictional model.

Frictional contact problems produce asymmetric stiffness matrices due to the existence of a tangential force. Using an asymmetric solver is more computationally expensive than a symmetric solver for each iteration. For this reason, ANSYS uses a symmetrization algorithm by which most frictional contact problems can be solved using solvers for symmetric

systems. Frictional effects and normal contact conditions are included through Lagrange multipliers or penalty terms. 

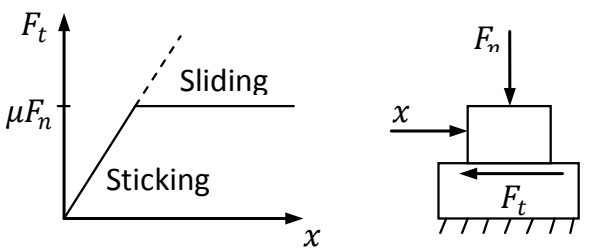

Fig. 1. Sticking and sliding zone in function of the normal load (comparable with a Jenkins or Maxwell-Slip element)

\section{INTERPRETATION OF THE RESULTS}

The FEA in Ansys Workbench consists of two bodies of each $100 \times 15 \times 15 \mathrm{~mm}$, making contact in one face. The upper body is constrained such that it can not move in two of its side planes and the lower body is constrained by two frictionless planes, as well a normal load and a remote displacement are applied to simulate the actual experiment. This simulation is performed for two materials, viz. PVC and $\mathrm{Al}$, and for 5 loads $(23,40,75,92,109 \mathrm{~N})$.

The results of the experimental data are used as an input for the simulations in Ansys Workbench. For each load a set of breakpoints is chosen with their corresponding friction coefficient. These breakpoints represent the displacement input for the lower body. For PVC, 10 equidistant points are used going from 0 till $20 \mu \mathrm{m}$ and for $\mathrm{Al} 6$ optimised points are used from 0 till $6 \mu \mathrm{m}$. This optimisation of point distribution is done based on the fact that for a higher curvature or lower radius of curvature for the non-linear behaviour, the density of points should be higher to have a more accurate linear piece wise representation of the original virgin curve. In this way a lower amount of breakpoints results into the same accuracy needing less simulation time.

The experimental results are used as a reference to compare the results of the FE simulations. As a first comparison the frictional force in function of the sliding distance is plot and compared with the friction force coming from the experiments, see Fig. 2. The frictional force coming from the simulations is slightly lower for all loads and have the same trend as the original virgin curve. This difference can be due to the lack of the memory effect, called the non-local memory in hysteresis behaviour, in the FE simulation. Each simulation, for each consecutive displacement, starts from zero until it found a numerical solution close to the input displacement coming from the 10 equidistant points for PVC and the 6 optimised points for Al. Improvements of the simulation consist of using the results of each simulation for each following displacement respectively, taking the local stiffness instead of the global stiffness into account. The simulation time can drastically be reduced by applying this alternative implementation method.

Another result of the simulation is the contact pressure, which is function of the tangential displacement or sliding distance. One would expect when no displacement is present that the pressure in the contact is equal to the applied load divided by the contact area. Since here, as in all contacts, the stiffness is normal load dependent for both the tangential and normal stiffness; the pressure in the contact has a similar hysteretic behaviour.

\section{CONCLUSION}

Using appropriate inputs and an optimised representation of the non-linear frictional behaviour, an accurate frictional contact simulation is obtained, taking the normal load and displacement dependency of the frictional behaviour into account through experimental correlation. The model can be improved in calculation time by using a step wise implementation starting from the previous results for each displacement respectively.
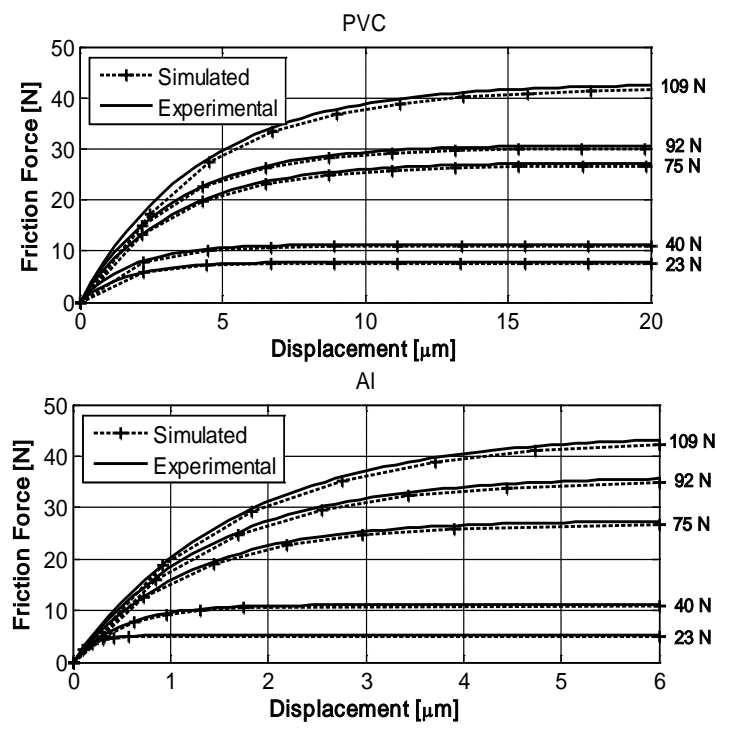

Fig. 2. Experimental and simulation results for the friction force in function of normal load and sliding distance

\section{ACKNOWLEDGEMENTS}

The work has been funded by the Sectoral Operational Programme Human Resources Development 2007-2013 of the Romanian Ministry of Labour, Family and Social Protection through the Financial Agreement POSDRU/88/1.5/S/60203 and POSDRU/88/1.5/S/61178. Thanks go to the Department of Mechanical Engineering from the K.U. Leuven, which made it possible to perform the experiments.

\section{REFERENCES}

Bengisu M.T. \& Akay A. (1997), Relation of dry friction to surface roughness, ASME J. Tribology, Vol. 119, January 1997, pp. 18-25

Blau, P. J., (2001), The Significance and Use of the Friction Coefficient, Tribol. Int., 34, pp. 585-591

Bowden, F.P. \& Tabor, D. (1964), The Friction and Lubrication of Solids, Part II, Clarendon, Oxford University Press, ISBN: 0198520263, UK

Greenwood, J.A. \& Tripp, J.H., (1967), The elastic contact of rough spheres, ASME J. Appl. Mech, 34, pp 153-160

Greenwood, J.A. \& Williamson, J.B.P., (1966), The contact of nominally flat surfaces, Proc. Roy. Soc. Lond. Vol. 295, pp. 300-319

Galin, L.A. (1980), Contact Problems in the Theory of Elasticity and Viscoelasticity (in Russian), Moscow: Nauka

Kalker, J.J. (1975), Aspects of contact mechanics, Proceedings of the symposium of the International Union of Theoretical and Applied Mechanics, pp.1-25

Karnopp D. (1985), Computer simulation of stick-slip friction in mechanical dynamic systems. Journal of Dynamic Systems, Measurement, and Control.(March 1985), Vol. 107 , pp. $100-103$

Kikuchi N. \& Oden, J. T. (1988), Contact Problems in Elasticity: A Study of Variational Inequalities and Finite Element Methods, SIAM, ISBN: 0-89871-468-0, Philadelphia

Muskhelishvili, N.I. (1966), Some Fundamental Problems of the Mathematical Theory of Elasticity, ISBN: 9001607012, Moscow: Nauka

Rivin, E.I. (1999), Stiffness and Damping in Mechanical Design, Marcel Dekker, Inc., ISBN 0-8247-1722-8, U.S.A 